\title{
Reconstrução cirúrgica da estenose cicatricial de vias biliares pós- colecistectomia
}

\author{
Surgical reconstruction of post-cholecistectomy cicatricial biliary stenosis
}

Aderivaldo Coelho de Andrade, TCBC-PI ${ }^{1}$

RES U M O

\begin{abstract}
Objetivo: Avaliar uma série de casos de estenose cicatricial de vias biliares pós-colecistectomia submetidos à reconstrução cirúrgica. Métodos: Foi realizado estudo retrospectivo de 27 pacientes submetidos à reconstrução cirúrgica da via biliar por estenose cicatricial. O tipo de colecistectomia que resultou na lesão, idade e sexo, sinais e sintomas, o momento do diagnóstico, se precoce ou tardio, presença de cirurgias prévias na tentativa de reconstruir a árvore biliar, classificação das estenoses, e tipo de operação empregada para o tratamento da injúria foram analisados. Resultados: Vinte e seis lesões ocorreram durante laparotomia e uma durante vídeolaparoscopia. Dezesseis pacientes (59\%) tiveram as lesões diagnosticadas no transoperatório ou nos primeiros dias de pós-operatório, sete $(26 \%)$ dos quais já submetidos à reoperação no hospital de origem, evoluindo mal; nove pacientes desse grupo (33\%) não tinham reoperação. Onze pacientes (41\%) apresentaram a forma clássica de estenose cicatricial, sem acidentes transoperatórios aparentes, com desenvolvimento de obstrução biliar tardia. Todos os pacientes foram submetidos à anastomose hepático-jejunal em "Y" de Roux, sendo que em dois casos os ductos hepáticos direito e esquerdo foram implantados separadamente na alça exclusa de jejuno. Vinte e seis pacientes $(96,3 \%)$ evoluíram bem inicialmente, um paciente teve fístula biliar e foi a óbito. Uma paciente com bom resultado inicial apresentou recidiva da anastomose, cirrose secundária e está aguardando transplante hepático. Conclusão: A maioria das lesões foi diagnosticada durante a colecistectomia ou nos primeiros dias de pós-operatório, sete pacientes já tinham sido operados na tentativa de reconstruir o trato biliar. A hepaticojejunostomia em "Y" de Roux empregada mostrou-se segura e efetiva em recanalizar a via biliar a curto e longo prazos.
\end{abstract}

Descritores: Colecistectomia. Constrição patológica. Ductos biliares. Complicações pós-operatórias. Ferimentos e lesões.

\section{INTRODUÇÃO}

pós a primeira colecistectomia realizada por
Langenbuch em 1882, na Alemanha, as complica-
ções biliares passaram a fazer parte da rotina dos cirurgi-
ões, sendo a estenose uma das mais graves e temidas des-
sas complicações. Concomitante ao avanço na área cirúr-
gica e ao número cada vez maior de colecistectomias rea-
lizadas, os problemas surgiram em número crescente. Fi-
nalmente, na década de 1980, considerava-se que o nú-
mero de lesões biliares pós-colecistectomias incidia em
$0,2 \%$ dos operados, sendo a estenose cicatricial um des-
ses problemas'.
Com a realização da primeira colecistectomia
vídeolaparoscópica, em 1987, por Mouret, na França, e
sua rápida e ampla divulgação, a ponto de em poucos
anos a vídeolaparoscopia ter substituído a laparotomia
no tratamento da colelitíase, o índice de injúrias biliares
pós-cirúrgicas aumentou ${ }^{1-6}$, sendo que nas primeiras es-
tatísticas o percentual era de $0,8 \%$, embora em alguns
relatos tenham sido apresentados resultados de até
$2,2 \%{ }^{1}$. Atualmente fala-se em $0,5 \%$, sendo que muitos autores apresentam resultados melhores, semelhantes ao da colecistectomia por laparotomia $(0,2 \%)$, entretanto há o viés estatístico nesses resultados, pois alguns pacientes não podem ser operados por vídeo ou têm sua operação convertida para a forma convencional, e assim o resultado real da vídeocirurgia não pode ser totalmente avaliado.

O desenvolvimento do reparo das lesões operatórias do trato biliar processou-se paralelamente com o progresso da cirurgia abdominal no final do Século XIX e por todo o Século $X X$, fazendo parte da rotina do cirurgião biliar em centros especializados, sendo que após o grande número de complicações relacionadas à vídeocirurgia houve um novo aumento no interesse sobre o tema, não só na maneira de se prevenir os acidentes, mas também na correção dos mesmos'.

O objetivo deste trabalho é estudar uma série de casos de estenoses cicatriciais de vias biliares, sua condução terapêutica e evolução, enfatizando a importância dessa grave complicação relacionada à colecistectomia.

Trabalho Realizado na Clínica Cirúrgica do Hospital Getúlio Vargas - Universidade Federal do Piauí - UFPI - PI-BR.

1. Cirurgião Geral do Hospital Getúlio Vargas - UFPI -PI-BR. 


\section{MÉTODOS}

Foram analisados retrospectivamente 27 pacientes submetidos à reconstrução cirúrgica da via biliar devido à estenose cicatricial pós-colecistectomia, no período compreendido entre setembro de 2001 e outubro de 2011, na Clínica Cirúrgica do Hospital Getúlio Vargas (HGV).

Todos os pacientes com sinais e sintomas de estenose biliar, estáveis clinicamente, foram incluídos no estudo. Foram excluídos os pacientes com quadro abdominal agudo, com peritonite, que necessitaram de tratamento cirúrgico de emergência. Foram analisados dados relativos ao tipo de operação que ocasionou a lesão, idade e sexo, sinais e sintomas, o momento do diagnóstico do estreitamento, se precoce ou tardio, presença de cirurgias prévias na tentativa de reconstruir a via biliar, classificação das estenoses e o tipo de operação empregada para o tratamento da injúria.

\section{RESULTADOS}

A colecistectomia por laparotomia foi a operação inicial em 26 pacientes, e a vídeolaparoscopia em um. $\mathrm{O}$ doente mais jovem tinha 20 anos de idade e o mais velho, 77; a média etária foi 41 anos.

Cinco pacientes eram homens $(11 \% \%)$ e 22 mulheres $(89 \%)$. Icterícia, colúria, acolia fecal e prurido estavam presentes em 26 casos. Uma das pacientes apresentava-se sem a clássica sintomatologia obstrutiva devido ao extravasamento da bile por uma fístula bíliocutânea.

O diagnóstico da lesão da via biliar principal foi feito no transoperatório em três pacientes e o cirurgião realizou a correção cirúrgica imediata. Após a boa evolução inicial todos os pacientes apresentaram icterícia, prurido e episódios de febre em um, três e seis meses, respectivamente. Treze pacientes apresentaram icterícia progressiva já nos primeiros dias de pós-operatório. Quatro foram reoperados no próprio Serviço de origem, sendo que três tiveram uma boa evolução, mas, após quatro meses, dois pacientes tornaram-se sintomáticos, com icterícia progressiva, prurido, colúria e acolia fecal. O outro ficou assintomático, mas, após um ano, passou a apresentar episódios de colangite, controlados com uso de antibióti- cos e dilatação da anastomose hepático-duodenal por via endoscópica, quando, finalmente, após 12 anos, desenvolveu estenose completa não dilatável. A paciente com hepático-jejunostomia em alça simples, não-exclusa, láterolateral, evoluiu mal desde o início, sendo encaminhada com quadro de fístula biliar. Os outros nove pacientes desse grupo foram encaminhados ao nosso Serviço sem tentativa de cirurgia reconstrutora. Entre aqueles com reoperação para correção da lesão biliar no hospital de origem, três apresentavam anastomoses hepático-duodenais, duas hepático-jejunostomias em " $Y$ " de Roux término-laterais e uma látero-lateral, bem como, uma hepático-jejunostomia em alça simples, não-exclusa, e todas evoluíram mal. Dentre esses havia uma paciente já submetida a quatro operações prévias, sendo a última uma hepático-duodenostomia, e outra paciente com três intervenções cirúrgicas prévias, sendo a última uma hepático-jejunostomia término-lateral em " $Y$ " de Roux (Tabela 1).

Do total de 27 pacientes, 11 tiveram a forma clássica de estenose cicatricial, onde não houve acidentes transoperatórios, entretanto, após período assintomático, que variou de três a 12 meses, apresentaram icterícia progressiva, colúria, acolia fecal e prurido, sugestivos de obstrução biliar. Em todos os 27 casos, diante da história clínica, foi realizada a colângio-ressonância que confirmou a estenose com dilatação à montante (Figura 1). Exames laboratoriais revelaram padrão colestático: hiperbilirrubinemia com predomínio da bilirrubina direta, níveis elevados de fosfatase alcalina e tempo de protrombina prolongado. Um dos pacientes foi admitido com cateter trans-hepático direito drenando a via biliar, sendo que o mesmo havia realizado colangiografia percutânea durante a colocação do cateter, contrastando somente o lobo direito, não havendo comunicação com o lobo esquerdo do fígado. A ressonância mostrou os ductos hepáticos direito e esquerdo sem comunicação (Bismuth IV).

Quanto à classificação idealizada por Bismuth: três pacientes (11\%) eram do tipo l; $15(55,5 \%)$ do tipo II; sete (26\%) tipo III e dois (7,5\%) tipo IV (Tabela 2$)$. Independente da gravidade da lesão sugerida pelo exame de imagem procedeu-se à cirurgia, sempre com o mesmo plano de reconstruir a via biliar anastomosando-a ao jejuno em "Y" de Roux. Todos os pacientes foram submetidos eletivamente à anastomose hepático-jejunal em " $Y$ " de

Tabela 1 - Tipos de reoperações realizadas no hospital de origem na tentativa de reconstruir a via biliar.

\begin{tabular}{lcc}
\hline Cirurgia realizada & $\mathbf{n}$ & $\%$ \\
\hline Nenhuma reoperação & 20 & 74 \\
Hepaticoduodenostomia & 3 & 11,1 \\
Hepaticojejunostomia em Y de Roux término-lateral & 2 & 7,5 \\
Hepaticojejunostomia em Y de Roux latero-lateral & 1 & 3,7 \\
Hepaticojejunostomia em alça simples & 1 & 3,7 \\
Total & 27 & 100 \\
\hline
\end{tabular}




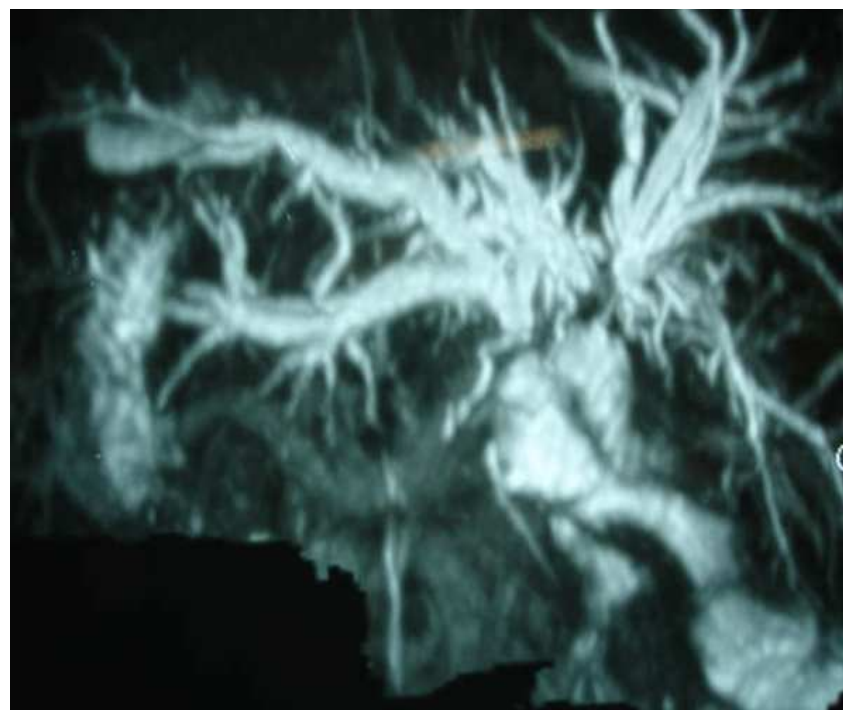

Figura 1 - Colângio-ressonância mostrando a via biliar intra-hepática, sem visualização da via extra-hepática. Esta paciente já tinha sido submetida a três cirurgias prévias, sendo a última uma hepaticojejunostomia em $Y$ de Roux que obstruiu, obrigando a uma reoperação.

Roux, sendo que em dois deles (7,5\%) os ductos hepáticos direito e esquerdo foram implantados separadamente no jejuno (Figura 2).

Entre os 27 doentes operados, 25 (92,6\%) tiveram boa evolução inicial, sem complicações precoces relacionadas às anastomoses. Duas pacientes tiveram evolução inicial problemática. Uma paciente apresentou fístula pós-operatória orientada pelo dreno, evoluindo bem, com fechamento espontâneo após dez dias; a outra teve evolução inicial ruim, apresentando fístula biliar de alto débito desde o pós-operatório imediato, tendo sido reoperada tardiamente no quarto dia, com reanastomose colângioentérica adequada, entretanto a paciente evoluiu com grave insuficiência respiratória decorrente de septicemia, com óbito subsequente no quinto dia pós-operatório. No geral 26 pacientes $(96,3 \%)$ tiveram alta hospitalar em boas condições. Três deles voltaram a apresentar icterícia obstrutiva em longo prazo. Um devido à obstrução por aderências na alça exclusa, realizou-se a lise de aderências, sendo que a anastomose foi avaliada e estava normal; o segundo, devi-

Tabela 2 - Distribuição dos casos (27 pacientes) segundo classificação de Bismuth para estenose de via biliar.

\begin{tabular}{lcc}
\hline Classificação de Bismuth & $\mathbf{n}$ & $\%$ \\
\hline Tipo I & 3 & 11 \\
Tipo II & 15 & 55,5 \\
Tipo III & 7 & 26 \\
Tipo IV & 2 & 7,5 \\
Total & 27 & 100 \\
\hline
\end{tabular}

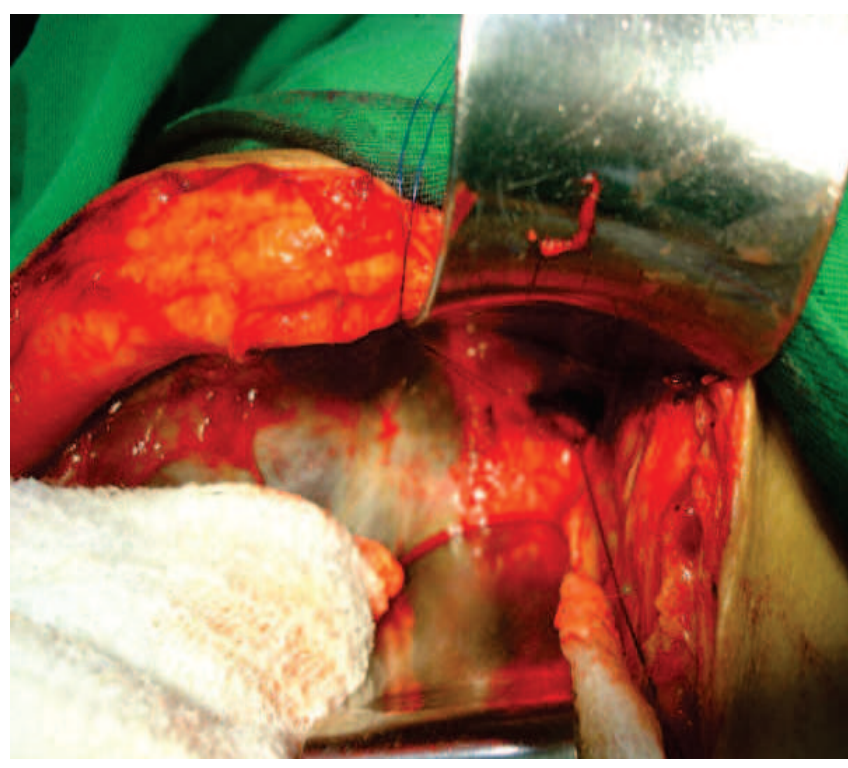

Figura 2 - Visão na cirurgia da pequena abertura do ducto hepático direito e da abertura ampliada do ducto hepático esquerdo.

do a uma semioclusão ileal por linfonodomegalias decorrente de uma paracoccidioidomicose abdominal, uma micose sistêmica presente no Norte do Brasil. À laparotomia foi realizada ileocolectomia direita com linfonodomegalia, pois havia a suspeita de carcinoma intestinal, sendo que a anastomose bíliodigestiva estava pérvia. O histopatológico revelou paracoccidioidomicose. A outra doente tinha passado de três operações prévias. Apresentou boa evolução inicial após reimplantação dos ductos hepáticos direito e esquerdo à alça de jejuno, com regressão da sintomatologia, entretanto, a partir do terceiro mês, apresentou episódios de icterícia, alternados com melhora clínica. Em virtude do agravamento do quadro foi submetida à dilatação da estenose por radiologia intervencionista, sem sucesso. Evoluiu com icterícia persistente, estando, no momento, clinicamente estável à custa de dreno biliar direito trans-hepático, aguardando transplante hepático. O caso em questão foi reavaliado e considerado que provavelmente esta paciente já fôra operada com quadro de cirrose biliar secundária em desenvolvimento, uma vez que foi admitida após seis meses do quadro de estenose estar bem estabelecido, tempo suficiente para o processo de cirrotização ter sido iniciado. Durante a reoperação os ductos foram identificados e reimplantados no jejuno, sendo que as anastomoses, especialmente a do ducto esquerdo, eram amplas, no entanto, após excelente evolução inicial, desenvolveu reestenose três meses após a cirurgia. Esta evolução é totalmente diferente dos outros pacientes, inclusive daqueles que a anastomose não ficou tão ampla. Acreditamos que o quadro fibrótico de cirrotização biliar secundária já tivesse em desenvolvimento e não reverteu após a drenagem adequada ter sido feita. Consequentemente a fibrose progrediu e levou à re-estenose das anastomoses, com agra- 
vamento do quadro de cirrose biliar e insuficiência hepática. Seis meses após a reconstrução biliar e consideradas falhas as tentativas de dilatação por radiologia foi encaminhada ao Serviço de Transplante Hepático.

No grupo de 26 pacientes, exceto por um caso, todos estão assintomáticos, variando o período entre a cirurgia reconstrutora da via biliar e o momento atual de um mês a dez anos.

\section{DISCUSSÃO}

A lesão iatrogênica é a principal causa de estenose benigna da via biliar (95\%)2 , e a colecistectomia é o procedimento mais comumente relacionado com estas lesões, podendo ocorrer também em operações envolvendo pâncreas, estômago e fígado. O dano ao hepatocolédoco pode ocorrer diretamente no transoperatório ou se apresentar tardiamente através da estenose cicatricial de uma via biliar intensamente inflamada ou desvascularizada no ato cirúrgico, assim como a fibrose cicatricial de uma anastomose previamente realizada. O tipo de lesão provocada no ato cirúrgico pode variar desde uma pequena laceração biliar, até grandes secções da via, com perda tecidual. Essas lesões operatórias podem ser detectadas imediatamente, observando-se diretamente o extravasamento de bile ou a ligadura e secção do hepatocolédoco, bem como, podem ser notadas no período pós-operatório com quadro clínico de coleperitôneo, seja com dor abdominal aguda, sugerindo peritonite biliar, ou somente com distensão abdominal pela ascite biliar. A icterícia progressiva é a forma de apresentação nos casos onde houve ligadura biliar, mas não há extravasamento de bile no abdome. O mesmo ocorre nos casos onde houve a estenose cicatricial tardia do colédoco, sem ter ocorrido acidentes transoperatórios, nestes pacientes os sintomas podem se apresentar semanas ou meses após com clínica predominante de colestase: icterícia, colúria, acolia fecal e prurido.

A causa do traumatismo biliar não mudou muito, valendo os mesmos motivos tanto para a laparotomia quanto para a laparoscopia: a inexperiência do cirurgião é sempre um fator importante, entretanto a anatomia biliar distorcida por anomalias congênitas e retrações cicatriciais devido a processos inflamatórios crônicos, edema local intenso nos quadros agudos, gordura excessiva na região do triângulo hepatocístico em obesos e alterações vasculares levando ao sangramento nos cirróticos, são todos fatores que dificultam a visualização da junção cístico-colédoco, aumentando o risco de acidentes, mesmo por cirurgiões experientes ${ }^{1}$. A cirurgia videolaparoscópica tem algumas particularidades que a tornam mais propensa ao trauma biliar, como a visão bidimensional em um monitor, diferente da visão tridimensional da cirurgia convencional, e também a impossibilidade de utilizar diretamente a mão para palpar a área cirúrgica ${ }^{1}$. Certamente esses fatores limitam o procedimento laparoscópico, havendo situações onde é impossível prosseguir a cirurgia e ocorre a conversão, e outras situações onde as tentativas exageradas em prosseguir com a operação sem as condições adequadas resultam em acidentes sobre a via biliar. Outra particularidade da laparoscopia é que as lesões são mais graves, mais proximais e com maior perda tecidual'.

Quanto à altura da obstrução, as estenoses biliares são agrupadas em níveis de um a quatro, conforme a classificação de Bismuth ${ }^{1,2}$. Esta se baseia na distância da lesão em relação à confluência dos hepáticos. Os estreitamentos grau I ficam a mais de $2 \mathrm{~cm}$ da confluência dos ductos hepáticos esquerdo e direito, os estreitamentos grau II ficam a menos de $2 \mathrm{~cm}$ da confluência, mas possuem um remanescente do ducto hepático comum, os estreitamentos grau III são coincidentes com a confluência, preservando-a, e os estreitamentos grau IV acometem acima da confluência dos ductos, desaparecendo a comunicação entre os lados direito e esquerdo do fígado.

Tanto o diagnóstico como o tratamento da estenose biliar sofreram mudanças graças aos avanços tecnológicos e ao melhor conhecimento sobre a doença adquiridos com o tempo. A ressonância nuclear magnética é utilizada no diagnóstico do paciente ictérico suspeito de estenose biliar ${ }^{2}$. Ela é não-invasiva, capaz de fazer um mapeamento adequado da via biliar e identificar o nível da obstrução; com isso, procedimentos invasivos, como as colangiografias trans-hepática e endoscópica, são menos utilizados quando a finalidade é puramente diagnóstica. Por outro lado, a abordagem terapêutica endoscópica e radiológica percutânea evoluíu bastante, sendo que muitas vezes a cirurgia é evitada graças às dilatações com balões e colocações de endopróteses utilizando os recursos da endoscopia e radiologia intervencionista, tanto como primeiro recurso terapêutico, como nas reabordagens após re-estenose das anastomoses bíliodigestivas realizadas para corrigir a estenose biliar ${ }^{1,6}$. Entretanto, esses procedimentos têm muitas limitações, sendo a principal delas a necessidade de haver um mínimo grau de continuidade na via biliar estenótica, o que não ocorre muitas vezes onde a obstrução é completa e há perda tecidual, assim, a cirurgia de reconstrução do trânsito biliar através da anastomose colângio-entérica continua sendo empregada na maioria dos casos ${ }^{1}$, inclusive porque centros especializados em cirurgia biliar são mais disseminados que os limitados centros de terapia endoscópica e radiológica. Embora haja várias maneiras de reconstruir a via biliar, atualmente a anastomose hepático-jejunal ou colédoco-jejunal em " $Y$ " de Roux é a mais aceita por apresentar os melhores resultados em longo prazo'

O prognóstico do tratamento cirúrgico depende de alguns fatores como o nível da lesão, o tempo percorrido entre a estenose e o tratamento, e se houve operações prévias $^{1,6}$. As lesões mais proximais são, tecnicamente, mais difíceis de reconstruir, apresentando pior prognóstico que os casos mais distais. Pacientes com estenose por período prolongado, admitidas tardiamente, muitas vezes já com 
dano hepático estabelecido, com fibrose cicatricial caracterizando o início e avanço da cirrose biliar secundária, certamente têm pior prognóstico após o tratamento cirúrgico, sendo maior a incidência de re-estenose, mesmo quando a anastomose foi tecnicamente bem feita ${ }^{5}$. As reoperações em vias biliares já reconstruídas têm duas particularidades: o paciente apresentou-se em situação de colestase e dano hepático mais de uma vez, além disso, há maior perda de via biliar e as estenoses são mais proximais e difíceis de reconstruir, o que torna o prognóstico cirúrgico pior. É importante enfatizar que a correção de lesão iatrogênica de vias biliares requer grande treinamento e perícia do cirurgião, podendo apresentar uma morbidade e mortalidade importantes, mesmo com equipes cirúrgicas experientes.

Com tratamento adequado, os resultados em longo prazo são bons ${ }^{1,4}$, mas, com manejo inadequado, a evolução é trágica para o doente, podendo levar às fístulas, peritonite, cirrose biliar, insuficiência hepática e óbito ${ }^{4}$.

No estudo apresentado são mostrados os bons resultados da anastomose bíliodigestiva em " $Y$ " de Roux em $92,6 \%$ dos pacientes, semelhantes aos melhores resultados obtidos na literatura ${ }^{1-6}$, enfatizando a falha no tratamento de um caso complexo, operado tardiamente, onde o paciente provavelmente já apresentava fibrose hepática irreversível e progressiva, que evoluiu e culminou com a obstrução das anastomoses separadas dos ductos hepáticos direito e esquerdo, adequadamente implantados no jejuno. O caso em questão, assim como outros dessa casuística, ensina que, tecnicamente, a correção de lesões proximais biliares em pacientes com múltiplas operações é plenamente possível por cirurgiões experientes, sendo que a colestase prolongada com dano ao fígado parece ser um dos fatores determinantes das re-estenoses e falha do tratamento cirúrgico bem feito. Isto levanta uma dúvida ao cirurgião biliar, considerando o bom estágio atual da cirurgia de transplante hepático: insistir em tentar reconstruir uma via biliar em paciente já com sinais clínicos, laboratoriais, radiológicos, endoscópicos e histopatológicos de colestase e cirrose biliar secundária ou encaminhar o paciente para um centro de transplante hepático ? Essa é uma questão extremamente importante, pois praticamente todas as grandes casuísticas ${ }^{1-}$ ${ }^{6}$ mostram um percentual variável de falha na terapêutica cirúrgica, com re-estenoses tardias. Por que a maioria dos pacientes se recupera bem e uma minoria volta a ter problemas, como é o nosso caso? É uma questão não completamente esclarecida. Certamente muitos desses pacientes foram operados já com cirrose biliar estabelecida, merecendo, portanto, que a possibilidade do transplante fosse avaliada logo, sem o paciente passar por todos os riscos de mais uma reanastomose biliar, com alta taxa de insucesso, distorcendo ainda mais a anatomia local e dificultando um futuro e, muitas vezes, inevitável transplante. Na paciente em questão não conseguimos definir previamente o problema. Não havia sinais clínicos claros, além disso, todos os primeiros 17 resultados cirúrgicos em longo prazo tinham sido bons, exceto por um mau resultado imediato de fístula devido à falha na técnica operatória.

A maioria das lesões foi diagnosticada durante a colecistectomia ou nos primeiros dias de pós-operatório, sete pacientes já tinham sido reoperados na tentativa de reconstruir o trato biliar. A hepaticojejunostomia em " $Y$ " de Roux mostrou-se segura e efetiva em recanalizar a via biliar a curto e longo prazos.

\title{
A B S T R A C T
}

\begin{abstract}
Objective: To evaluate a series of cases of cicatricial stenosis of the biliary tract after cholecystectomy undergoing surgical reconstruction. Methods: We conducted a retrospective study with 27 patients who underwent surgical reconstruction of the biliary tree for cicatricial stenosis. We analyzed the type of cholecystectomy that resulted in injury, age, gender, signs and symptoms, time of diagnosis, early or late, presence of previous surgery in an attempt to reconstruct the biliary tree, classification of stenosis and type of operation used for treatment of the injury. Results: Twenty-six injuries occurred during a laparotomy and one during laparoscopy. Sixteen (59\%) lesions were diagnosed intraoperatively or within the first postoperative day, seven (26\%) havinh been submitted to reoperation at the local hospital, with poor results; nine patients in this group (33\%) had no reoperation. Eleven patients (41\%) had the classic form of cicatricial stenosis, without apparent intraoperative accidents and late development of biliary obstruction. All patients underwent Roux-en-Y hepatic-jejunal anastomosis; in two cases the right and left hepatic ducts were implanted separately in the excluded jejunal loop. Twenty-six patients (96.3\%) had no early complications; one patient had biliary fistula and died. One patient presented with stenosis recurrence, secondary cirrhosis and is awaiting liver transplantation. Conclusion: Most injuries were diagnosed during cholecystectomy within the first postoperative days; seven patients had been reoperated in an attempt to reconstruct the biliary tract. Roux-en-Y hepaticojejunostomy proved safe and effective in draining the bile duct in the short and long term.
\end{abstract}

Key words: Cholecistectomy. Constriction, pathologic. Bile ducts. Postoperative complications. Wounds and injuries.

\section{REFERENCIAS}

1. Rossi RL. Revisão das lesões do trato biliar. Clin cir Am Norte. 1994;74(4):785-94.
2. Marson AC, Mali Jr J, Oliveira RG, Valezi AC, Brito EM, Libos Jr Farid. Tratamento cirúrgico das estenoses da via biliar. Rev Col Bras Cir. 2004;31(4):224-7.

3. Hajinasrollah E, Salehi N, Mohsen H, Ghazal H. Bile duct injury of laparoscopic cholecystectomy. Gastroenterology and Hepatology from Bed to Bench. 2008;1(2):95-8. 
4. Jablonska B, Lampe P, Olakowski M, Gorka Z, Lekstan A, Gruszka T. Hepaticojejunostomy vs end-to-end biliary reconstructions in the treatment of iatrogenic bile duct injuries. J Gastrointest Surg. 2009;13(6):1084-93.

5. Pottakkat B, Vijayahari R, Prassad KV, Sikora SS, Behari A, Singh RK, Kumar A, Saxena R, Kapoor VK. Surgical management of patients with post-cholecystectomy benign biliary stricture complicated by atrophy-hypertrophy complex of the liver. HPB. 2009:11(2):125-9.

6. Nuzzo G, Giuliante F, Giovannini I, Murazio M, D'Acapito F, Ardito F, Vellone M, Gauzolino R, Costamagna G, Di Stasi C. Advantages of multidisciplinary management of bile duct injuries occurring during cholecystectomy. Am J Surg. 2008;195(6):763-9.
Recebido em 03/06/2011

Aceito para publicação em 08/08/2011

Conflito de interesse: nenhum

Fonte de financiamento: nenhum

\section{Como citar este artigo:}

Andrade AC. Reconstrução cirúrgica da estenose cicatricial de vias biliares pós-colescistectomia. Rev Col Bras Cir. [periódico na Internet] 2012; 39(2). Disponível em URL: http://www.scielo.br/rcbc

Endereço para correspondência:

Aderivaldo Coelho de Andrade

E-mail: domderi@hotmail.com 